\title{
Warum eine Risikoabschätzung und Grenzwertsetzung für Mikrokunststoffe in der aquatischen Umwelt problematisch ist
}

\author{
Maria Fürhacker \\ Online publiziert: 7. August 2020 \\ (C) Der/die Autor(en) 2020
}

Zusammenfassung Kunststoffe sind allgegenwärtig und werden in allen aquatischen Umweltkompartimenten den Meeren, in Flüssen, an Stränden, den Sedimenten und in der gesamten Wassersäule und auch innerhalb von Biota gefunden. Durch dessen Zerfall entstehen kleinere Bruchstücke, die unter den Begriff Mikroplastik (MP) fallen. Ein besonderes Problem in der Diskussion stellt die Definition von Mikroplastik dar. Die obere Grenze von $5 \mathrm{~mm}$ ist von den EU-Mitgliedsstaaten und vielen internationalen Organisationen akzeptiert, aber die Definition einer unteren Grenze ist sehr verschieden, z.B. umfasst sie bei ECHA $1 \mathrm{~nm}$ oder bei EFSA $100 \mathrm{~nm}$ oder $1 \mu \mathrm{m}$. Ziel dieses Artikels ist es, verschiedene Informationen und Kenntnisse aber auch offene Fragen über MP in der Umwelt zusammenzuführen und die komplexen Zusammenhänge in Hinblick auf MP, dessen Definition, Untersuchungsmethodik und die damit verbundenen Probleme für eine Risikoabschätzung und Grenzwertsetzung aufzuzeigen. Neben der Definition, sind auch die Methoden zur Probenahme und Analyse und Zuordnung zu Partikelgrößen und die Erfassung der relevanten Wirkungen noch nicht standardisiert. Aktuell erfolgt die quantitative Bestimmung von MP hauptsächlich über mikroskopische, spektroskopische oder thermoanalytische Methoden, wobei es nur mit spektroskopischen Verfahren möglich ist, die Partikelanzahl, die Partikelgröße und das Material zu bestimmen, die aber nur bis zu Größen

Ao.Univ.-Prof. DI Dr. M. Fürhacker ( $₫)$

Department für Wasser,

Atmosphäre und Umwelt,

Institut für Siedlungswasserbau,

Industriewasserwirtschaft und

Gewässerschutz, Universität

für Bodenkultur Wien,

Muthgasse 18, 1190 Wien, Österreich

maria.fuerhacker@boku.ac.at von $20 \mu \mathrm{m}$ (FTIR) bzw. $1 \mu \mathrm{m}$ (Raman) verlässliche Daten liefern. Die potenziellen toxischen Einflüsse einer Exposition gegenüber MP können vielfältig sein und von Fütterungsstörungen, Verletzungen und Geschwüren, Verstopfungen des Verdauungstrakts, bis hin zu verminderter Reproduktionsleistung, Störungen des Energiestoffwechsels, bzw. Veränderungen der Leberphysiologie reichen. Die wenigen verfügbaren Daten zu den Wirkungen zeigen, dass Partikel im Nanobereich signifikant wirksamer sind als solche im $\mu \mathrm{m} \mathrm{Be}$ reich. Über indirekte Gefahren durch die Polymere selbst über Restmonomere oder Polymeradditive oder an MP adsorbierte Umweltschadstoffe, gibt es wenige Studien, die den spezifischen Einfluss der Kontamination von der Wirkung des MP trennen. Da die Expositionsdaten mit den Wirkdaten, wegen der fehlenden Größenklassen, nicht ohne weiters vergleichbar sind, ist eine Risikobewertung und Grenzwertsetzung schwierig und auch fraglich, weil das Risikomanagement in der Umwelt problematisch ist. Obwohl verschiedene Experten, die vorläufige Risikoabschätzungen durchgeführt haben, ein konkretes Risiko durch MP (in der untersuchten Größe zumeist $>300 \mu \mathrm{m}$ ) im aquatischen Bereich ausschließen, wählt die ECHA und die UNEP einen Vorsorgeansatz in dem sie die Verwendung von MP (Definition $1 \mathrm{~nm}-5 \mathrm{~mm}$ ) stark einschränkt und MP auf die Liste $\mathrm{XV}$ (substances of very high concern) setzt. Dies wird damit begründet, dass MP persistent ist, leicht aufgenommen wird, dadurch in die Nahrungskette gelangt und potenzielle Auswirkungen auf die menschliche Gesundheit haben kann und es unmöglich ist MP aus der Umwelt zu entfernen.

Schlüsselwörter Mikroplastik .

Definition von MP .

Bestimmungsmethoden . ökotoxikologische Effekte

\section{Why a risk assessment and the setting of limits for micro-plastics in the aquatic environment is problematic}

Abstract Plastics are omnipresent and are found in all aquatic environmental compartments of the oceans, in rivers, on beaches, in the sediments and in the entire water column and also within biota. When it decays, smaller fragments are formed which finally end up as microplastics. A particular problem in the discussion is the definition of microplastics. The upper limit of $5 \mathrm{~mm}$ is accepted by the EU member states and many international organizations, but the definition of a lower limit is very different, e.g. it includes $1 \mathrm{~nm}$ for ECHA or $100 \mathrm{~nm}$ or $1 \mu \mathrm{m}$ for EFSA. The aim of this article is to compile various information and knowledge as well as open questions about MP in the environment and to show the complex relationships with regard to MP, its definition, investigation methodologies and the associated problems for a risk assessment and standard setting.

In addition to the definition, the methods for sampling and analysis and assignment to particle sizes and the investigation of the relevant effects are not yet standardized. Currently, the quantitative determination of MP is mainly carried out using microscopic, spectroscopic or thermoanalytical methods, whereby it is only possible with spectroscopic methods to determine the number of particles, the particle size and the material, but reliable data are only observed up to sizes of $20 \mu \mathrm{m}$ (FTIR) or $1 \mu \mathrm{m}$ (Raman). The potential toxic effects of exposure to MP can be diverse and range from feeding disorders, injuries and ulcers, constipation of the digestive tract to reduced reproductive performance, disorders of energy metabolism, or changes in liver physiology. The few data available on the effects show that 
particles in the nano range are significantly more effective than those in the $\mu \mathrm{m}$ range. There are few studies on the indirect dangers posed by the polymers themselves via residual monomers or polymer additives or environmental pollutants adsorbed to $\mathrm{MP}$, which separate the specific influence of contamination from the effect of MP itself. Since the exposure data are not readily comparable with the impact data due to the lack of size classes, risk assessment and setting limits is difficult and also questionable because risk management in the environment is problematic. Although various experts who have carried out preliminary risk assessments negate a specific risk from MP (mostly $>300 \mu \mathrm{m}$ ) for the size examined in the aquatic environment, ECHA and UNEP choose a precautionary approach for the use MP (definition $1 \mathrm{~nm}-5 \mathrm{~mm}$ ) and place MP on List XV (substances of very high concern). This is due to the fact that MP is persistent, easily taken up and thereby entering the food chain and have potential negative effects on human health and it is impossible to remove MP from the environment.

Keywords Microplastics - Definition of microplastics - Detection methods Ecotoxicological effects

\section{Einleitung}

Das Thema Kunststoffverschmutzung in der Umwelt wurde aufgrund der mannigfaltigen Verwendung von Kunststoffen zu einem Hauptpunkt in der öffentlichen Diskussion, und da die Verwendung und Produktion sehr hoch ist, wird erwartet, dass das Problem auch in nächster Zeit aktuell bleibt. Dabei kommt dem Mikroplastik (MP), sowohl was primäres als auch sekundäres $\mathrm{MP}$ betrifft, eine besondere Rolle zu. MP ist aber nicht immer nur das unerwünschte Nebenprodukt, in vielen Anwendungen hat es auch eine wichtige vorteilhafte Funktion, wobei Substanz, Zustand, Morphologie und Dimension für seine Eigenschaften wichtig sind.

MP kommt in allen aquatischen Umweltbereichen, an Stränden, im Meer und in Oberflächengewässern, in der gesamten Wassersäule und innerhalb von Biota vor. Die Verteilung von MP in der Umwelt ist schwer zu erfassen, weil sie von meteorologischen, zeitlichen und geografischen Faktoren und von den MP-Eigenschaften wie Dichte,
Form, Größe, Adsorption von Chemikalien und Biofouling sowie von Umgebungsbedingungen wie Wasserdichte, Wind, Strömungen und Wellen beeinflusst wird. Dies führt aufgrund der relativ geringen Größe und großen Oberfläche zur Akkumulation über einen bestimmten Zeitraum in verschiedenen ökologischen Kompartimenten, was die Probenahme und auch die Aussagekraft der Ergebnisse beeinträchtigen kann. MP wird in allen Kompartimenten gefunden und obwohl nur wenige Daten zur Wirkung auf aquatische Biozönosen vorliegen, die Übertragung auf höhere trophische Ebenen und die Abschätzung Auswirkungen von Mikroplastik auf die natürliche Umwelt und die menschliche Gesundheit äußerst schwierig ist, geht man davon aus, dass die Emissionen von (Mikro-) Kunststoffen in die Umwelt irreversible negative Auswirkungen haben.

Ziel dieses Artikels ist es, verschiedene Informationen und Kenntnisse aber auch offene Fragen über MP in der Umwelt zusammenzuführen und die komplexen Zusammenhänge in Hinblick auf MP, dessen Definition, Untersuchungsmethodik und die damit verbundenen Probleme für eine Risikoabschätzung aufzuzeigen. Diese Kenntnisse sind wichtig für das Verständnis künftiger bzw. geplanter Maßnahmen und für die Entwicklung anwendbarer Managementinstrumente und -richtlinien zur Bewältigung dieses Themas.

\section{Definition von Mikroplastik}

Mikroplastik (MP) ist jener Teil des Gesamtplastiks dessen Partikelgrößen zwischen Makroplastik (>5 mm) und Nanoplastik (Partikel kleiner als $1 \mu \mathrm{m}$ bzw. kleiner als $100 \mathrm{~nm}$ ), bei jedenfalls $<5 \mathrm{~mm}$ liegt, wobei die untere Größenbegrenzung noch immer nicht konsistent definiert ist.

Man unterscheidet zwischen primärem (Typ A und B) und sekundärem MP. Unter dem primären MP-Typ A versteht man MP-Partikel, die in dieser Größe produziert wurden und in Produkten z. B. Peelings, Zahnpasten oder Putzpasten, inclusive Luftstrahlmedien, eingesetzt werden. Primäres MP Typ B entsteht bei der Nutzung, z. B. durch Abrieb wie Fasern aus Kleidung oder Partikel aus Reifen (Hann et al. 2018). Sekundäres MP wird während oder nach Gebrauch von Kunststoffen durch Fragmentierung größerer Plas- tikstücke in immer kleinere Teilchen unbeabsichtigt freigesetzt.

Die Definition von MP, wie sie in vielen EU-Mitgliedsstaaten, aber auch international verwendet wird, ist breit und umfasst synthetische Polymerteilchen von weniger als $5 \mathrm{~mm}$, die in Wasser unlöslich sind und einem (Bio-)Abbau widerstehen.

Die Grundlage für die Obergrenze einer Partikelgröße von MP $5 \mathrm{~mm}$ war eine pragmatische Entscheidung, die auf der Annahme beruhte, dass diese Partikel leicht von Biota (wie Wirbellosen, Fischen, Meeresreptilien, Vögel und Walen (entweder direkt oder durch Sekundärvergiftung) und auch zu einer Exposition des Menschen führen (durch Nahrung oder Wasser, das MP enthält)) aufgenommen werden können, und solche Partikel andere Arten von Bedrohungen darstellen als größere Kunststoffgegenstände (GESAMP 2015). Allerdings wurde die untere Grenze für die Partikelgrößen noch nicht ausreichend definiert, wodurch auch die Studienergebnisse sehr schwer vergleichbar sind.

Nach Definition der Europäischen Lebensmittelagentur (European Food Safety Agency EFSA) (EFSA 2016a) haben Mikrokunststoffe eine Größe von 0,1 bis 5000 Mikrometer $(\mu \mathrm{m})$. Nanokunststoffe messen zwischen 0,001 und $0,1 \mu \mathrm{m}$ ( 1 bis $100 \mathrm{~nm}$ ).

Die Europäische Umweltagentur (European Chemicals Agency ECHA) (ECHA 2018) diskutierte noch eine untere Größenbeschränkung für MP von $1 \mu \mathrm{m}$, will sie aber nicht beschränken, weil kleinere Partikel in der Regel reaktiver sind und auch leichter biologische Membranen passieren können. Allerdings räumt ECHA (2018) ein, dass eine höhere Größenbeschränkung für die Praktikabilität und Durchsetzbarkeit einer vorgeschlagenen Beschränkung wichtig sein kann und möchte die Angemessenheit einer unteren Größengrenze im Bereich von Nanometer (zwischen 1 und $100 \mathrm{~nm}$ ) bis Mikrometer prüfen.

Wie schwierig schlussendlich eine Definition von MP sowohl in Hinblick auf Substanz als auch in Hinblick auf die Größe ist, zeigt das Dokument der ECHA (2019), das in Ermangelung einer Definition von „Kunststoff“ unter REACH als Ausgangspunkt für eine regulatorische Definition von „Mikroplastik“, folgende regulatorische Definitionen vorschlägt: 
- „Mikroplastik“ bezeichnet ein Material, das aus festen polymerhaltigen Partikeln besteht, denen möglicherweise Additive oder andere Substanzen zugesetzt wurden, und bei denen $\geq 1$ Gew.-\% Partikel vorhanden sind (i) alle Abmessungen $1 \mathrm{~nm} \leq x \leq 5 \mathrm{~mm}$ oder (ii) für Fasern eine Länge von $3 \mathrm{~nm} \leq x \leq 15 \mathrm{~mm}$ und ein Verhältnis von Länge zu Durchmesser von $>3$.

- Polymere, die in der Natur vorkommen und nicht chemisch modifiziert wurden (außer durch Hydrolyse), sind ausgeschlossen, ebenso wie Polymere, die (biologisch) abbaubar sind.

Dadurch inkludiert diese MP-Materialdefinition sehr viele unterschiedliche synthetisch hergestellte Polymere, wie dies in dem aktuellen Beschränkungsvorschlag der Europäischen Chemikalienagentur (ECHA) für MP als Produktzusatz (ECHA 2019) diskutiert wird. In diesem Vorschlag wird der Größenbereich von MP auf $1 \mathrm{~nm}$ bis $5 \mathrm{~mm}$ ausgedehnt. Allerdings ist es auch wichtig, die Dimensionen $\mathrm{zu}$ bestimmen. Die Beschränkung auf eine Dimension würde sämtliche Kunststoffe, die in einer Dimension $<5 \mathrm{~mm}$ sind, also auch Folien oder Plastiksackerl, in die MPDefinition einschließen.

\section{Verwendung von MP}

Durch diese sehr umfassende Definition sind nicht nur MP in Kosmetikprodukten oder Polymere in Waschund Wartungsprodukten, sondern auch sehr viele Polymere erfasst, die häufig in landwirtschaftlichen und gartenbaulichen Produkten verwendet werden, um Samen während der Keimung zu schützen, die Freisetzung von Düngemitteln und Pflanzenschutzmitteln zu kontrollieren und zu verzögern, sowie als Düngemittelzusätze eingesetzt werden. MP findet sich, aufgrund seiner wasserunlöslichen, inerten Eigenschaften in Lebensmitteln, Nahrungsergänzungsmitteln und auch in vielen Arzneimitteln und Medizinprodukten als Träger und im medizinischen Bereich, z. B. in in-vitro-Diagnostika wie in Immunoassays, und wird in der Nuklearmedizin für die diagnostische Bildgebung verwendet. MP wird in Farben und Lacken, in der Wasser- und Abwassertechnik (z. B. als Polymere) und auch im Öl- und Gassektor verwendet, z. B. zur Gewährleistung der Integrität der Ölquelle, als Korrosionsinhibitor, zur Maximierung der Ölund Gasgewinnung oder zur Redukti- on des Energieverbrauchs. Die größten Emissionen sind mit der Verwendung in Demulgatoren, Antischaummitteln, Korrosions- und Wachsinhibitoren verbunden (ECHA 2019).

\section{Bestimmungsmethoden für MP}

Bei der Bestimmung von MP aus verschiedenen Medien bestehen offene Fragen in Hinblick auf die Probenahme und Quantifizierung der MP-Partikel, sowohl was die Probenaufbereitung, den Einfluss der Anreicherung von MP aus wässrigen Proben und festen Proben als auch die Bestimmung der Größe und des Materials angeht.

Methodik und Menge des Probenmaterials beeinflussen die Repräsentativität der Ergebnisse. Deshalb werden oft große Wassermengen in situ durch Verwendung von Netzen, Sieben oder Pumpen reduziert. Allerdings hat die gewählte Maschenweite einen großen Einfluss. Vermaire et al. (2017) zeigten, dass mittels Nylonnetz $(100 \mu \mathrm{m})$ fast hundertmal höhere Konzentrationen an MP ermittelt wurden als mit Mantanetzen $(333 \mu \mathrm{m}), 0,1$ bzw. $0,00135 \mathrm{MP} / \mathrm{L}$. Die Poren- oder Maschengröße bestimmt die geringste Größe der nachgewiesenen MP-Partikel; kleine Porenoder Maschengrößen können jedoch $\mathrm{zu}$ einer schnellen Verstopfung durch organische und mineralische Stoffe führen.

Die quantitative Bestimmung von MP erfolgt derzeit hauptsächlich über mikroskopische, spektroskopische Verfahren oder thermoanalytische Methoden. Die rein bildgebenden Verfahren (z.B. Mikroskopie, Licht-/Elektronenmikroskopie) ebenso wie reine Partikelzählverfahren (z. B. über Licht-/ Laserstreuung) haben den Nachteil, dass sie keine Information über die chemische Zusammensetzung des Materials liefern. Zudem besteht die Gefahr der Fehlinterpretation von MP, weil die Ermittlung von falsch positiven wie auch falsch negativen Ergebnissen möglich ist. Durch Färbung mit z.B. Nilrot können die MP-Partikel im Fluoreszenzmikroskop sichtbar gemacht werden, allerdings kann Nilrot auch die natürlichen organischen Stoffe färben, deshalb sollte diese Färbemethode nicht alleine angewendet werden (Li et al. 2018). Bei den thermoanalytischen Verfahren, die einen Gesamtgehalt in Milligramm Kunststoff pro Kilogramm oder Liter ermitteln können, werden vorzugsweise Pyrolyse-
GC-MS (Gas-Chromatographie-Massenspektrometrie) sowie TED-GC-MS (Thermo-Extraktion-Desorption GasChromatographie-Massenspektrometrie) eingesetzt. Die spektroskopischen Verfahren, erlauben die Bestimmung der Partikelanzahl einzelner Kunststoffe in Stück pro Kilogramm oder Liter sowie die Partikelgröße und können das Material identifizieren. Zu den spektroskopischen Verfahren zählen RamanSpektroskopie und FT-IR Spektroskopie (Fourier Transformation Infrarotspektroskopie). Die Verwendung von spektroskopischen Methoden erlaubt die Identifikation der verschiedenen Kunststofftypen. Insbesondere Kombinationen der spektroskopischen Methoden mit bildgebenden Verfahren sind vorteilhaft, z.B. Raman-Mikroskopie oder FT-IR Mikroskopie. Die untere Grenze der Bestimmung mittels Raman liegt bei ca. 0,5-1 $\mu \mathrm{m}$, während die Bestimmung mittels FT-IR mit $20 \mu \mathrm{m}$ begrenzt ist (Käppler et al. 2016). Auch wenn MP-Partikel bis $1 \mu \mathrm{m}$ detektiert werden können, identifizieren nur wenige Umweltstudien Partikel $<50 \mu \mathrm{m}$ aufgrund methodischer Einschränkungen (Hidalgo-Ruz et al. 2012; Imhof et al. 2016). Prata et al. (2019) untersuchten 40 Studien zu MP in Wasser und Sediment wobei $50 \%$ FT-IR-basierte Methoden, $32,5 \%$ visuelle Inspektion, $10 \%$ Raman-Spektroskopie, und jeweils 2,5\% Elektronenmikroskopie, Farbstoffe und Gaschromatographie-Massenspektroskopie verwendeten.

Eine Reihe von Faktoren kann die Repräsentativität von Daten $\mathrm{zu} \mathrm{MP}$ beeinflussen, darunter räumliche und zeitliche Variabilität, Partikelarten, Methoden der Probenahme, Größenauswahl, Extraktionsmethoden, Trennung, Verdauung, Reduktion von Kreuzkontaminationen, Methoden der Identifizierung, Charakterisierung und Quantifizierung von Massenproben. Bei der Probenahme sowie der Aufbereitung der Proben für die Analytik muss gewährleistet sein, dass es zu keiner Veränderung, Zerstörung oder Verlusten der $\mathrm{zu}$ untersuchenden Kunststoffe kommt. Zugleich bedarf es Vorkehrungen, die eine zusätzliche Verunreinigung der Probe mit Kunststoffen (z. B. durch Laborequipment, synthetische Bekleidungsfasern, Staub) während der Aufbereitung und Analyse verhindern bzw. minimieren. Es wird empfohlen, zur Bewertung einer möglichen MPKontamination im Labor entsprechende Kontrollproben mitzuführen. Da 
Probenahme-, Extraktions-, Nachweismethoden und -techniken weltweit entwickelt werden, ist eine Harmonisierung und Standardisierung der Techniken und Protokolle dringend erforderlich, wobei kleine Partikel z. B. mit einer Größe $<150 \mu \mathrm{m}$ bzw. von 20 bis $30 \mu \mathrm{m}$, aber auch $<1 \mu \mathrm{m}$ und Nanopartikeln charakterisiert und quantifiziert werden müssen, um vergleichbare Ergebnisse, insbesondere zu kleinem MP $(<1 \mathrm{~mm})$, und in weiterer Folge für die Berechnung von Mengenströmen für MP in der Umwelt, zu erzielen und um das Risiko reproduzierbar einschätzen zu können.

Die Ergebnisse werden normalerweise als Gesamt-MP pro Probeneinheit (z. B. pro L in Wasser) ausgedrückt und sollten detaillierte Angaben $\mathrm{zu}$ Größenklassen, Farbe und Form (z.B. Faser, Partikel, Fragment) und ob Polymerkautschuk enthalten ist oder nicht, enthalten. Allerdings wird oft auch die Masse angegeben, wodurch es schwierig ist verschiedene Literaturstellen miteinander zu vergleichen, weil eine Umrechnung insofern problematisch ist, als ein Partikel mit einem Radius von $500 \mu \mathrm{m}$ dasselbe Volumen bzw. Gewicht hat wie $10^{6}$ Partikel mit einem Radius von $5 \mu \mathrm{m}$ Partikel bzw. $10^{9}$ mit einem Radius von $0,5 \mu \mathrm{m}$.

Für die Abschätzung des Risikos ist es notwendig die Messergebnisse in Größenklassen anzugeben, weil die Wirkungen im Wesentlichen von der Größe abhängig sind. Vom deutschen BMBF (2018) wird eine Klassierung von MP-Ergebnissen in Größenklassen z.B. $5000-1000 \mu \mathrm{m},<1000-500 \mu \mathrm{m}$, $<500-100 \mu \mathrm{m},<100-50 \mu \mathrm{m},<50-10 \mu \mathrm{m}$, $<10-5 \mu \mathrm{m},<5-1 \mu \mathrm{m}$ vorgeschlagen. Es gibt jedoch derzeit keine Analysenmethode, die eine so breite Vielfalt von Größenbereichen, wie von ECHA (2019) gefordert, von Mikro- bis Nanoplastik in Umweltproben und von Substanzen quantitativ nachweisen kann. Für das Monitoring der Umweltbelastung mit MP und der Überprüfung von etwaigen Grenzwerten werden darüber hinaus Analysensysteme benötigt, die automatisiert werden können und dabei rasche und valide Ergebnisse zu einem günstigen Preis für den Endkunden liefern.

\section{Voraussetzungen für eine Risikoabschätzung}

Eine klassische Risikoabschätzung (ECHA 2016) muss die Gefährdung ermitteln, eine Dosis-Wirkungs-Bezie- hung erstellen, die Exposition erfassen und bewerten und das Risiko charakterisieren. Die Ermittlung der schädlichen Wirkungen, umfasst Informationen zu inhärenten Eigenschaften des Stoffes, die Bestimmung von Derived No-Effect-Levels (DNEL-Werten) für die einschlägigen Endpunkte der menschlichen Gesundheit und die Bestimmung von Predicted No-effect-Effect Levels (PNEC-Werten) für die Umweltendpunkte (ECHA 2011).

Für die Frage der MP Partikel stellt sich diese Aufgabe sehr komplex dar, weil die mit MP verbundenen Gefahren physikalische, chemische und biologische Aspekte haben, und ihre möglichen Auswirkungen auf das Ökosystem artenabhängig sind, sowie räumlich und zeitlich variieren. Auf der Grundlage einer Kombination aus Beobachtungen, Laborexperimenten und Expertenmeinungen könnten Gefahrentabellen für eine Reihe von Ökosystemkomponenten und MP-Eigenschaften erstellt werden. Es ist jedoch sehr schwierig Eintrittswahrscheinlichkeiten zuzuordnen. Von der GESAMP (2016) wird die Entwicklung eines einfachen Risikobewertungsrahmens vorgeschlagen, der den Prozess der Systemprüfung formalisiert, mögliche Konsequenzen beschreibt und die Eintrittswahrscheinlichkeit vorhersagt.

Ein Problem bei der Interpretation der Studien ergibt sich daraus, dass in den Studien häufig weder eine untere noch eine obere Größenbeschränkung oder nur die angestrebte Größenklasse angegeben wird. Die Studien, die kleinere Partikel erfassen, wie einige der Studien mit Wasser in Flaschen und Leitungswasser, ergeben im Allgemeinen höhere Partikelanzahlkonzentrationen. Die erste Frage, die geklärt werden muss ist: Welche Partikelgrößen beziehen wir in die Betrachtung mit ein?

\subsection{Exposition}

Internationale Studien schätzen die Anzahl der MP-Teilchen in den Meeren zwischen 5 und 50 Billionen Teilchen ( $>330 \mu \mathrm{m}$ bzw. $280 \mu \mathrm{m}$ ), bei einer Masse von 32.000 bis 236.000 Tonnen, wobei die Probenahmen üblicherweise mit Schleppnetzen gemacht werden und die Daten sich auf die obersten $10(-15 \mathrm{~m})$ beziehen (Eriksen et al. 2014). Zwei Studien zur Abschätzung der Häufigkeit von MP auf der Meeresoberfläche gehen von einer 100-fach geringeren Zahl von kleinen MP-Teilchen $(<1 \mathrm{~mm})$ im Vergleich zu größeren MP (1 bis $5 \mathrm{~mm}$ ) aus (Cózar et al. 2014; Eriksen et al. 2014).

Koelmans et al. (2019) untersuchten 50 Studien, deren Datenqualität zum Teil sehr schlecht war und von denen nur vier eine positive Bewertung für alle vorgeschlagenen Qualitätskriterien erhielten (Koelmans et al. 2019). Die MP-Konzentrationen, angegeben als Anzahl an Partikel, umspannten insgesamt zehn Größenordnungen $\left(1 \times 10^{-2}\right.$ bis $10^{8} \mathrm{Partikel} / \mathrm{m}^{3}$ ) je nach Einzelprobe und Wasserart. In Oberflächengewässern decken MP-Konzentrationen $>300 \mu \mathrm{m}$ einen weiten Konzentrationsbereich ab, ungefähr $1 \times 10^{-3}$ bis 10 Partikel/L; für Leitungswasser (Bereich $1 \times 10^{-4}$ bis 100 Partikel/L) werden oft höhere Zahlen gemessen, weil sehr oft Partikel ab $1 \mu \mathrm{m}$ oder ab $100 \mu \mathrm{m}$ erfasst werden.

Eine österreichische Studie (Hohenblum et al. 2015) untersuchte MP $(>500 \mu \mathrm{m})$ in der fließenden Welle der Donau und fand mittlere Jahresfrachten in Aschach $<14 \mathrm{t}$ pro Jahr und in Hainburg $<41$ t pro Jahr. Wenn man eine mittlere Durchflussmenge in Wien rund $2000 \mathrm{~m}^{3}$ pro Sekunde berücksichtigt, dann liegen die Konzentrationen bei ca $0,0007 \mathrm{mg} / \mathrm{L}$ und damit weit unter der Konzentration von anderen Spurenstoffen. Diese Studie untersuchte auch 30 Fische aus der Donau im Bereich der Messstelle Hainburg auf das Vorkommen von Plastik im Verdauungstrakt. Es konnten in den untersuchten Fischen keine Plastikteilchen identifiziert werden.

In einer Vielzahl anderer Studien wurde die Aufnahme von MP in Organismen unterschiedlicher trophischer Ebenen dokumentiert, wie Filtrierern, z. B. der Muschel Mytilus edulis (Browne et al. 2008), Wirbellosen (Murray und Cowie 2011) oder kommerziell gefangenen Fischarten (Lusher et al. 2013; Miranda und Carvalho-Souza 2016; Neves et al. 2015).

MP hat eine Größe und oft auch Farbe, die wie charakteristischen Beuteorganismen ähnelt, was das Potenzial der Aufnahme durch Wasserorganismen erhöht (Ory et al. 2017). MP kann auf unterschiedliche Weise in das Nahrungsnetz aquatischer Spezies gelangen:

- direkt durch Aufnahme von MP,

- durch zufällige Aufnahme,

- Verwechslung mit Nahrung oder 
- durch Aufnahme einer Beutetierart, die bereits MP enthält (Wright et al. 2013).

Lusher et al. (2013) fanden z.B. in zehn Arten von pelagischen und demersalen Fischen aus dem Ärmelkanal in 36,5\% MP, während Neves et al. (2015) von 26 Meeresspezies an der portugiesischen Küste in 19,8\% der Fische Plastikabfälle im Verdauungstrakt fanden. Eine Untersuchung von 2233 Fischen aus zwei Flussmündungen (von 69 Arten) in Brasilien zeigte, dass 9\% der Individuen (24 Arten) unabhängig von der Fischgröße, MP (durchschnittlich 1,06 $\pm 0,30$ Partikel/Gesamtfisch) im Darminhalt hatten (Vendel et al. 2017).

Eher kritisch $\mathrm{zu}$ sehen ist MP in Nanogröße, weil diese Partikel hauptsächlich über Endozytose über die Zellmembran transportiert werden und somit als zellulärer Vektor (Trojanisches Pferd) für andere Chemikalien oder Nano-Additive dienen können. Was das Vorkommen von MP in Nanogröße in der Umwelt betrifft, sind die Konzentrationen aufgrund methodischer Einschränkungen unbekannt. Ohne ein Verständnis der Menge in der Umwelt ist es jedoch schwierig, eine umweltrelevante Dosis für Experimente oder Risikobewertungen zu identifizieren.

In Bezug auf die Lebensmittelsicherheit hängen die möglichen Auswirkungen von MP auf die menschliche Gesundheit von der Exposition durch die Nahrung und das Trinkwasser ab. Die angegebenen Konzentrationen sind im Wesentlichen von der Bestimmungsmethode abhängig. In einer eigenen BOKU-Studie (Vymetal 2016) wurden Oberflächenwässer, Uferfiltrate und abgefüllte Flaschenwässer untersucht. Während in Oberflächenwässern MP $(>30 \mu \mathrm{m})$ gefunden wurde, konnten in den Uferfiltraten keine MP-Partikel ( $>30 \mu \mathrm{m}$, Probenvolumen $>100 \mathrm{~L}$ ) detektiert werden. In den abgefüllten Wässern reichten die Ergebnisse von 0 bis 10 Partikel/L. Auch eine norwegische Studie kommt zu dem Schluss, dass in Norwegen MP im Trinkwasser nicht nachgewiesen wurde (Nachweisgrenze 4,1 Partikel/L) (Uhl und Eftekhardadkhah 2018). Im Gegensatz dazu fanden Kosuth et al. (2018) in $81 \%$ der 159 weltweit bezogenen Leitungswasserund Flaschenwasserproben anthropogene Partikel. Die meisten dieser Partikel waren Fasern $(98,3 \%)$ mit einer Länge zwischen 0,1 und $5 \mathrm{~mm}$. Der Bereich betrug 0 bis 61 Partikel/L, mit einem Gesamtmittel von 5,45 Parti$\mathrm{kel} / \mathrm{L}$ (Filterporenweite 2,5 $\mathrm{m}$, Probenvolumen 500-600 ml). In dieser Studie wurden nicht angefärbte Partikel mikroskopisch erfasst und als MP ausgewiesen. Eine Verifikation mittels Raman oder FT-IR wurde nicht vorgenommen. Mason et al. (2018) fanden in $93 \%$ von insgesamt 259 untersuchten Flaschenwasserproben durchschnittlich 10,4 MP-Partikel/L $>100 \mu \mathrm{m}$. Bei der Hälfte dieser Partikel wurde mittels FTIR-Spektroskopie bestätigt, dass sie Polymere sind, wobei Polypropylen, das für Flaschenverschlüsse verwendet wird, am häufigsten war (54\%). Kleinere MP-Partikel $(6,5-100 \mu \mathrm{m})$ wurden nach Adsorption von Nilrot-Farbstoff spektroskopisch durch Fluoreszenz, ohne Verifikation, erfasst. Es wurden durchschnittlich 325 MP-Partikel/L $(6,5-100 \mu \mathrm{m})$ in den Flaschen gefunden (MP-Kontaminationsbereich von 0 bis über 10.000 MP-Partikeln/L), wobei $95 \%$ der Partikel zwischen 6,5 und $100 \mu \mathrm{m}$ groß waren. Die Daten legen nahe, dass die Kontamination zumindest teilweise von der Verpackung und/ oder dem Abfüllprozess selbst herrührt.

Von der EFSA (2016b) sollte das Risiko für den Menschen durch MP in Meeresfrüchten abgeschätzt werden. Die Konzentration von MP in marinen Spezies wurde im gesamten Verdauungstrakt bestimmt. In Fisch liegt die durchschnittliche Anzahl zwischen 1 und 7 Partikel/g. In Garnelen werden durchschnittlich 0,75 Partikel/g und Muscheln 0,2-4 Partikel (Medianwert)/g gefunden (EFSA 2016b).

\subsection{Wirkungen von MP in der aquatischen Umwelt und auf den Menschen}

Damit ein Risiko abgeleitet werden kann, muss zuerst eine Exposition erfolgen. Das Ausmaß des Risikos ist üblicherweise proportional zur Wirksamkeit des Stoffes und zum Ausmaß der Exposition. Es stellt sich die Frage, welche adversen Wirkungen durch welche MP-Partikelgrößen ausgelöst, bzw. welche Organ- oder Zellfunktionen gestört werden. Einige Xenobiotika verursachen Toxizitäten, indem sie an Proteine (strukturell, Enzyme), DNA (Mutationen) oder Lipide binden und diese schädigen oder mit Sauerstoff freie Radikale bilden und so Lipide, Proteine und DNA schädigen. Für all diese Reaktionen ist die sogenannte biologisch effektive Dosis entscheidend, die von der Toxikokinetik, d.h. von Adsorption, Distribution, Metabolismus und Exkretion und auch von der Toxikodynamik, der Reaktion im System selbst, abhängig ist.

Die potenziellen toxischen Einflüsse einer Exposition gegenüber MP können vielfältig sein und von Fütterungsstörungen, Verletzungen und Geschwüren, Verstopfungen des Verdauungstrakts, die zu Pseudosättigung, Hunger und körperlicher Beeinträchtigung führen können, bis hin zu verminderter Reproduktionsleistung, Störungen des Energiestoffwechsels bzw. Veränderungen der Leberphysiologie reichen (Cole et al. 2013, 2015). Marine Organismen werden durch die Nahrungsaufnahme (einschließlich Filtration, aktive Beweidung und Sedimentaufnahme) und über die Kiemen (Belüftung) MP ausgesetzt. Aufnahme, Anreicherung und Exkretion und Wirkung von MP hängen von der Partikelgröße ab.

Faktoren für das entstehende Risiko sind zusätzlich:

- Anzahl der Partikel;

- Art der Teilchen (z.B. Polymertyp, Größe, Form und Alter);

- Expositionsdauer;

- Konzentration und Art der mit dem Kunststoff verbundenen Bestandteile und Verunreinigungen; und

- Physiologie und Lebensgeschichte des Organismus (GESAMP 2016).

All diese Informationen sind für eine Wirkanalyse notwendig.

In den meisten Labor- und Feldstudien wird nur die Aufnahme von MP in den Darm, nicht aber die physiologische Wirkung betrachtet.

Sehr kleine MP-Teilchen (Größenbereich $\mathrm{nm}$ bis einige wenige $\mu \mathrm{m}$ ) können durch Zellmembranen treten und in Zellen und Geweben eine Immunantwort oder Entzündungen hervorrufen. In Laborstudien konnten letale Wirkungen vor allem durch kleine Partikelgrößen $(<1 \mu \mathrm{m})$ beobachtet werden. Bei extrem hohen nanoskaligen MP-Konzentrationen von 1,4 bis $40 \mathrm{mg} / \mathrm{ml}$ wurde die Photosynthese bei den Algenspezies (Chlorella und Scenedesmus) gehemmt, möglicherweise auch aufgrund der physikalischen Blockade von Licht und Luft, außerdem wurde auch die Produktion reaktiver Sauerstoffspezies erhöht, was auf einen Zustand von oxidativen Stress hinweist (Bhattacharya et al. 2010). Daten über die Translokation in den Blutkreislauf oder ins Gewebe sind sehr selten. Browne et al. (2008) 
waren die ersten, die mit Muscheln Mytilus edulis zeigten, dass kleine Polystyrol Mikrokügelchen ( 3 und $10 \mu \mathrm{m}$; 40 Teilchen $/ \mathrm{ml}$ ) innerhalb von 3 Tagen, das Potenzial haben, sich aus dem Verdauungstrakt in die Hämolymphe zu verlagern. MP kann im Laborexperiment bei Copepoden die Mortalität erhöhen und die Fruchtbarkeit verringern (Lee et al. 2013), MP kann sessilen Organismen wie Muscheln auch den Filtermechanismus stören und eine Entzündungsreaktion auslösen (MP $>0$ bis $80 \mu \mathrm{m} ; 2,5 \mathrm{~g} / \mathrm{L}$ ) (von Moos et al. 2012). Besseling et al. (2013) stellten eine positive Beziehung zwischen der MP-Konzentration im Sediment (MP $0,1,10$, und $100 \mathrm{~g} \mathrm{MP} / \mathrm{L}$ Sediment, Größenbereich 0,4-1,3 mm) und der Aufnahme sowie dem Gewichtsverlust durch den Wattwurm fest. Trotz dieser physischen Auswirkungen konnte keine Akkumulation im Verdauungstrakt während des Experiments (28 Tage) festgestellt werden.

MP kann auch Vektor für die synergistische und/oder antagonistische Wirkung anderer hydrophober organischer Verunreinigungen sein; die Wirkung kann sich auf unterschiedlichen trophischen Ebenen entfalten (Anbumani und Kakkar 2018). Indirekte Gefahren wie Karzinogenese oder endokrine Störungen (Oehlmann et al. 2009; Talsness et al. 2009) sind mit Umweltschadstoffen wie z.B. Organochlor-Pestiziden (OCPs), polychlorierten Biphenylen (PCBs) und polycyclischen aromatischen Kohlenwasserstoffen (PAHs), bromierten oder fluorierten Flammschutzmitteln, polybromierten Diphenylethern (PBDEs), Hexabromcyclodecan (HBCDs) und PerfluoralkylSäuren (PFAAs) sowie Zusatzstoffen (Bisphenol A (BPA), Nonylphenol (NP) und Octylphenol (OP)) verbunden, die an MP-Partikeln adsorbieren, nachdem sie an die Umwelt abgegeben wurden (Ziccardi et al. 2016). (Öko-) toxikologische Gefahren können auch von den Polymeren selbst über Restmonomere oder Polymeradditive (z. B. Stabilisatoren, Weichmacher, Flammschutzmittel) ausgehen. Bei non-target Screening-Analysen von Chemikalien wurden insgesamt 231 bis 251 organische Verbindungen auf Kunststoffabfällen festgestellt, darunter Kohlenwasserstoffe, UV-Stabilisatoren, Antioxidantien, Weichmacher, Flammschutzmittel, Schmiermittel, Zwischenprodukte und Verbindungen für Farbstoffe und Tinten (Gauquie et al. 2015; Rani et al.
2015). Die Konzentrationen der Verunreinigungen sind im Hafenbereich oft um Größenordnungen höher als im offenen Meer. Negative Effekte durch adsorbierte Chemikalien hängen vom biologischen Transfer ab, der durch äußere Faktoren wie Art, Größe und Menge des Kunststoffs, Konzentration und Eigenschaften (z.B. Hydrophobizität) der Chemikalien auf dem Kunststoff, der Ökologie (Art des Stoffwechsels und insbesondere der Trophie) und Physiologie des Tiers und der Verweilzeit im Tier beeinflusst wird. In Laborstudien mit verunreinigten Kunststoffen wurden nachteilige Wirkungen allein durch den Kunststoff nachgewiesen, die sich verstärkten, wenn die Organismen der Mischung aus Kunststoff mit sorbierten Chemikalien ausgesetzt wurden (Browne et al. 2013; Rochman et al. 2013), was darauf hindeutet, dass die Kombination aus Kunststoffabfällen und prioritären Spurenstoffen eine Mehrfachbelastung darstellen kann.

Koelmans et al. (2016) weisen darauf hin, dass Feldstudien auch mit den Problemen der Mehrfachverursachung, des Mangels an Gradienten und der Variabilität der Umwelt zu kämpfen haben, was ihre Verwendung zum Nachweis des Beitrags der MP-Aufnahme zur Bioakkumulation einschränkt.

Die tatsächlichen Auswirkungen von MP auf die aquatischen Ökosysteme sind wegen der limitierten Daten über die toxikologischen Risiken von MP für Wasserorganismen noch weitgehend spekulativ und die toxischen Wirkungen sind inkonsistent. Die Studien spiegeln zumeist auch nicht die subletalen chronischen Expositionsszenarien wider, denen die Biozönosen in der Umwelt ausgesetzt sind. Die Mehrzahl der veröffentlichten Wirkungen umfasst subletale Reaktionen von Organismen auf MP mit Tieren in Laborversuchen unter extremen Bedingungen. Im Vergleich zu Laboruntersuchungen gibt es nur sehr wenige direkte Hinweise auf physikalische Einwirkungen von MP in der Natur und wenig Beweise aus Feldstudien, die negative Effekte unter natürlichen Bedingungen belegen (GESAMP 2016).

Jedenfalls ist die Übertragung von MP-induzierten nachteiligen Auswirkungen auf verschiedenen trophischen Ebenen des marinen Nahrungsnetzes von hohem Interesse (Farrell und Nelson 2013; Setala et al. 2014).

Über die Auswirkungen von Mikround speziell sehr kleinen MP-Teilchen (d.h. Nanokunststoffpartikel $<1 \mu \mathrm{m}$ in mindestens einer ihrer Dimensionen) auf die menschliche Gesundheit weiß man sehr wenig (Eerkes-Medrano et al. 2015; Van Cauwenberghe und Janssen 2014; Bouwmeester et al. 2015; GESAMP 2015). Lt. EFSA (2016b) fehlen Angaben zur Toxizität und Toxikokinetik von MP und Nanokunststoff für eine Risikobewertung beim Menschen. Experimente mit Nagetieren zeigen, dass MP $>1 \mu \mathrm{m}$ über die Lymphe in den Blutkreislauf gelangen kann, aber nicht tief in die Organe eindringen kann, während Nanokunststoffe $(<100 \mathrm{~nm})$ alle Organe einschließlich Plazenta und Gehirn erreichen und durchdringen können (Bouwmeester et al. 2015; GESAMP 2015). MP wurde auch in menschlichen Stuhlproben gefunden (Schwabl et al. 2018); für die menschliche Gesundheit werden deshalb eher Kunststoffadditive wie Phthalate, Bisphenol A, bromierte Flammschutzmittel, Triclosan und Organozinnverbindungen als relevant betrachtet.

Es wird auch diskutiert, dass MPKrankheitserreger transportieren, was ein Gesundheitsrisiko für Meeresorganismen und Menschen darstellen kann. MP kann auch die Populationsgröße sowie die Ausbreitung von Arten beeinflussen. Zukünftige Arbeiten sind erforderlich, um Ausmaß und Auswirkungen dieses Effekts zu verstehen (GESAMP 2016).

\section{Vorläufige Risikoabschätzung}

Everaert et al. (2018) analysierten das Risiko durch MP für die Meeresumwelt, basierend auf dem von der European Chemicals Agency (2016) beschriebenen Verfahren, wobei die Umweltkonzentrationen auf der globalen Produktion (aus der Vergangenheit (1950-2016), der Gegenwart (2018) und der Zukunft (2100)) und Umweltparametern für Kunststoff modelliert und die Toxizitätsdaten aus der Literatur gesammelt wurden. Für die Exposition im Jahr 2100 werden 9,6 bis 48,8 MPPartikel $/ \mathrm{m}^{3}$ (3 Größenklassen $<500 \mu \mathrm{m}$, 0,5-1 mm, 1-5 mm) angenommen. Das sind 50-mal so viele wie jetzt, wobei eine sichere Konzentration bei 6650 MPPartikel $/ \mathrm{m}^{3}$ abgeleitet wurde. Auf dieser globalen Skala stellten die Autoren bis 2100 kein unmittelbares Risiko in Meeresgewässern, weder für die Wassersäule noch fürs Sediment, fest. Auch die EurEau (2019) hat sich mit dieser Thematik beschäftigt. Auch wenn im 
Meer selbst bis 2100 kein Risiko zu erwarten ist, werden die Strände allerdings bei Annahme einer gleichbleibenden aktuellen Kunststoffproduktion bereits 2040 unsicher. Das MP-Problem in Ozeanen scheint hauptsächlich auf „hot spots“ beschränkt zu sein (EurEau 2019). Burns and Boxall (2018) veröffentlichten eine Risikobewertung zu MP in Süß- und Meerwasser. Im Vergleich der gemessenen Umweltkonzentrationen mit den in der Literatur angegebenen Öko-Toxizitätswerten scheint MP kein Risiko für die aquatischen Organismen darzustellen.

Von Adam et al. (2019) wurde eine Risikoabschätzung für Frischwasser durchgeführt. Bevorzugte ökotoxikologische Endpunkte waren Überleben, Wachstum, Fortpflanzung und Veränderungen wesentlicher Stoffwechselprozesse wie Photosynthese, die auf Ökosystemebene am bedeutendsten sind. Die PNEC-Konzentration wurde als fünftes Perzentil der probabilistischen Speziessensitivitätsverteilung berechnet, basierend auf 53 Werten aus 14 Süßwasserspezies ergab sich ein Wert von $7,4 \times 10^{5}$ Teilchen $/ \mathrm{m}^{3}$ (25. und 75. Quantil von 6,1 $\times 10^{5}$ bzw. $1,3 \times 10^{6} \mathrm{Partikel} / \mathrm{m}^{3}$ ). Die meisten gemessenen MP-Konzentrationen lagen zwischen $10^{-2}$ und $10^{4}$ Teilchen $/ \mathrm{m}^{3}$. In Europa wurde mehr als die Hälfte der Konzentrationen zwischen 0,1 und $10 \mathrm{Partikel} / \mathrm{m}^{3}$ gemessen. Die höchsten Konzentrationen von bis zu $5,2 \times 10^{5} \mathrm{Partikel} / \mathrm{m}^{3}$ wurden in Asien festgestellt. Allerdings wurden unterschiedliche Grenzgrößen von 0,45 bis $500 \mu \mathrm{m}$ verwendet. Mehr als die Hälfte der Proben wurde mit Cutoff-Größen $>100 \mu \mathrm{m}$ gesammelt, aber mehr als ein Viertel wurde durch Poren von $<50 \mu \mathrm{m}$ filtriert, wobei in Asien kleinere Partikelgrößen erfasst wurden. Zur Ermittlung des Risikoquotienten (RCR) wurde die Expositions- durch die PNEC-Wahrscheinlichkeitsverteilung dividiert. Es wurden RCRs von 1,3 $\times 10^{-6}$ für Nordamerika, $3,3 \times 10^{-6}$ für Europa und $4,6 \times 10^{-3}$ für Asien berechnet. Die Risikocharakterisierung ergab für Europa und Nordamerika $<\mathbf{1}$, d.h. hier ist derzeit kein Risiko zu erwarten. Die RCRs zeigten, dass ökologische Risiken in Asien, wo 0,4\% der RCR-Werte über 1 lagen, nicht vollständig ausgeschlossen werden können (Adam et al. 2019).

Die Auswertung der vorhandenen Literatur durch die EFSA ergab, dass die Daten über Auftreten, Toxizität und
Verbleib von MP für eine vollständige Risikobewertung unzureichend sind und dass Nanokunststoffe besondere Aufmerksamkeit verlangen. Für den Menschen schätzte die EFSA, ausgehend von den wenigen vorliegenden Daten, dass eine Portion Muscheln (225g) $7 \mu \mathrm{g}$ Mikrokunststoffe enthalten könnte. Doch selbst wenn diese Menge an Kunststoff die höchsten je gemessenen Konzentrationen an PCB oder BPA enthielte, um zwei Beispiele zu nennen, wäre dies nur ein kleiner Beitrag zur Gesamtexposition gegenüber diesen Stoffen: Die PCB-Exposition würde sich um weniger als 0,01\% erhöhen, die BPA-Exposition um weniger als zwei Prozent. Hierbei handelt es sich jedoch um ein Worst-Case-Szenario (EFSA 2016b).

Der Wissenschaftsrat für die Politik der Europäischen Akademiker (The Science Advice for Policy by European Academics SAPEA) schrieb kürzlich in einem Bericht für die Europäische Kommission: „Die besten verfügbaren Erkenntnisse legen nahe, dass MP und Nanokunststoff kein weit verbreitetes Risiko für Mensch und Umwelt darstellen, außer in kleinen Nischen." SAPEA weist jedoch auch auf Wissenslücken und das Risiko hin, dass sich die Situation ändern könnte (EurEau 2019).

\section{Maßnahmen}

Auch wenn verschiedene vorläufige Risikoabschätzungen zumindest für $\mathrm{MP}$ keine alarmierenden Daten ergeben haben, gibt MP trotzdem - angesichts seiner Persistenz in der Umwelt und eines potenziellen Zerfalls zu sehr kleinen MP-Partikeln, sowie die damit verbundenen möglichen Auswirkungen auf die Ökosysteme - zunehmend Anlass zur Sorge und steht seit einiger Zeit ganz oben auf der Tagesordnung der EntscheidungsträgerInnen.

Die EU hat mit der EU-Plastikstrategie (EU 2018) bereits Schritte unternommen, z. B. Maßnahmen zur Senkung des Verbrauchs von Plastiksackerl, Förderung höherer Recyclingquoten und bessere Abfallsammelsysteme, Wiederverwendung von Verpackungen, Verringerung des Verpackungsbedarfs für Flaschenwasser, Förderung eines umweltfreundlichen öffentlichen Beschaffungswesens und Überwachung und Reduzierung von Meeresmüll. Durch Maßnahmen der Industrie, die den Zugang zu EU-Märkten und internationalen Märkten erhalten möchte, konnte bereits zwischen 2012 und 2017 eine substanzielle MP-Substitution bei bestimmten kosmetischen Produkten von mehr als $97,5 \%$ erzielt werden; für MP in medizinischen Lebensmitteln und Nahrungsergänzungsmitteln sowie für medizinische Anwendungen sind derzeit keine Alternativen verfügbar (ECHA 2019). Die ECHA (2019) prüft die Notwendigkeit einer REACHBeschränkung für die absichtliche Verwendung von „Mikroplastik“-Partikeln. Durch die Zuordnung von MP zur Liste $\mathbf{X V}$, die auf den potenziellen Umweltund Gesundheitsrisiken und der Persistenz begründet sind, soll die Verwendung von MP wesentlich eingeschränkt werden. Es bleibt aber die Option, von den Schlussfolgerungen in ECHA (2019) abzuweichen, falls diese durch weitere Daten zu Exposition und Risiken von MP oder anderen sozioökonomischen Faktoren gerechtfertigt sind.

\section{Offene Fragen}

Die wichtigsten offenen Fragen betreffen ganz Grundsätzliches, ohne deren Klärung eine Diskussion schwierig ist, z. B. die Vereinheitlichung von Begriffen und Methoden zur Probenahme und Analyse, die Untersuchung von MP in verschiedenen Umweltbereichen und Zuordnung zu Partikelgrößen (insbesondere im Nanobereich) und die Erfassung der relevanten Wirkungen auf höheren Ebenen der biologischen Organisation (z. B. Population, Spezies, Artengesellschaft, Ökosystem). Offen sind auch Erkenntnisse zum Transport von MP durch Nahrungsnetze, ebenso wie das Schicksal und Risiko durch sorbierte Chemikalien und Zusatzstoffe und adsorbierte Mikroorganismen weiter fraglich ist. Jedenfalls ist es momentan nicht möglich die Wirkungen und Umwelteinflüsse von MP zu interpretieren, da weder die verwendeten Assays noch die Analysenmethoden einheitlich und adäquat sind.

Die EFSA (2016a) empfiehlt daher, die Analysemethoden für MP weiterzuentwickeln und für Nanokunststoffe zu entwickeln und zu standardisieren, um das Vorhandensein, die Identität und die Menge in Lebensmitteln aber auch in der Umwelt zu bestimmen. Zusätzlich sind Untersuchungen zur Toxizität und vor allem zur Toxikokinetik auch der kleinsten Größenklassen erforderlich.

Obwohl in Hinblick auf MP sehr viele Studien bereits durchgeführt wurden 
und werden, fehlt es an Daten, evidenzbasierte Entscheidungen in $\mathrm{Be}$ zug auf die ökologische Exposition, die Auswirkungen und die Risiken von Mikroplastik zu unterstützen. Die Daten zur Exposition erlauben keinen sinnvollen Vergleich mit Wirkdaten, weil toxische Wirkungen auch im unteren Größenbereich ( $\mathrm{nm}$ bis wenige $\mu \mathrm{m}$ ) ermittelt wurden, während sich die Expositionsdaten wegen physikalischer Grenzen der Probenahme und Bestimmungsmethoden zumeist auf Größen von $50-300 \mu \mathrm{m}$ beziehen und meist nicht in Klassen unterteilt sind. Viele dieser Probleme treten auch bei technischen Nanopartikeln (ENPs) und chemischen Gemischen auf. Aus diesem Grund schlagen Syberg et al. (2015) vor, Erkenntnisse aus der Nanoforschung auf MP anzuwenden.

\section{Schlussfolgerungen}

Es zeigt sich, dass durch die Limitierungen bei Probenahme und Analyse und der fehlenden Standardisierung der Angabe der Untersuchungsergebnisse in Größenklassen, die Exposition (PEC) und wegen der Komplexität der Wirkung von MP unterschiedlicher Größenklassen auf verschiedene Spezi- es auch diese schwer abschätzbar und kein PNEC-Wert ableitbar ist. Für eine Risikoabschätzung sind zusätzlich Informationen über Formen, Materialien und Verunreinigungen notwendig. Erst danach könnte man an eine Festlegung von Grenzwerten denken, um negative physikalische, chemische und ökologische Auswirkungen in verschiedenen Lebensräumen, die auf eine Kontamination von MP in der Umwelt zurückzuführen sind, zu verhindern.

Von den EntscheidungsträgerInnen der UNEP (2016) und auch von der ECHA (2019) wird wegen der aktuellen Unsicherheiten beim Verständnis der Wirkungen, die als irreversibel angenommen werden - und weil es nicht möglich ist, MP, das einmal in der Umwelt ist, zu entfernen -, ein Weg gewählt, der nach dem Vorsorgegedanken handelt und bereits die Verwendung von MP in der umfassenden ECHADefinition (ECHA 2019), massiv einschränkt.

Funding Open access funding provided by University of Natural Resources and Life Sciences Vienna (BOKU).

Open Access Dieser Artikel wird unter der Creative Commons Namensnen- nung 4.0 International Lizenz veröffentlicht, welche die Nutzung, Vervielfältigung, Bearbeitung, Verbreitung und Wiedergabe in jeglichem Medium und Format erlaubt, sofern Sie den/die ursprünglichen Autor(en) und die Quelle ordnungsgemäß nennen, einen Link zur Creative Commons Lizenz beifügen und angeben, ob Änderungen vorgenommen wurden.

Die in diesem Artikel enthaltenen Bilder und sonstiges Drittmaterial unterliegen ebenfalls der genannten Creative Commons Lizenz, sofern sich aus der Abbildungslegende nichts anderes ergibt. Sofern das betreffende Material nicht unter der genannten Creative Commons Lizenz steht und die betreffende Handlung nicht nach gesetzlichen Vorschriften erlaubt ist, ist für die oben aufgeführten Weiterverwendungen des Materials die Einwilligung des jeweiligen Rechteinhabers einzuholen.

Weitere Details zur Lizenz entnehmen Sie bitte der Lizenzinformation auf http://creativecommons.org/licenses/ by/4.0/deed.de.
Adam, V., Yang, T. and Nowack, B. (2019): Toward an ecotoxicological risk assessment of microplastics: Comparison of available hazard and exposure data in freshwaters. Environmental Toxicology and Chemistry 38(2), 436-447.

Anbumani, S., Kakkar, P. (2018): Ecotoxicological effects of microplastics on biota: a review. En viron Sci Pollut Res 25, 14373-14396. https://doi org/10.1007/s11356-018-1999-x

Besseling, E., Wegne, A., Foekema, E.M., Vandenheuvel-Greve, M.U., Koelmans, A.A. (2013) Effects of microplastics on fitness and PCB bioaccumulation by the lugworm Arenicola marina (L.): Environ Sci Technol 42:5026-5031

Bhattacharya, P., Lin, S., Turner, J.P., Ke, P.C (2010): Physical adsorption of charged plastic nanoparticles affects algal photosynthesis. J Phys Chem C 114:16556-16561

BMBF (2018): Diskussionspapier - Mikroplastik-Analytik. Bundesministerium für Bildung und Forschung, Deutschland. https:// bmbf-plastik.de/sites/default/files/2018-10/ Diskussionspapier\%20Mikroplastik-Analytik. pdf. (Zugriff 03.07.2020)

Bouwmeester, H., P. C. Hollman and R. J. Peters (2015): Potential health impact of environmentally released micro- and nanoplastics in the human food production chain: Experiences from nanotoxicology. Environmental Science \& Technology 49(15): 8932-8947.

Browne, M.A., A. Dissanayake, T. S. Galloway,

D. M. Lowe and R. C. Thompson (2008): Ingested microscopic plastic translocates to the circulatory system of the mussel, Mytilus edu- lis (L.). Environmental Science \& Technology 42(13): 5026-5031.

Browne, M.A., S. J. Niven, T. S. Galloway, S. J. Rowland and R. C. Thompson (2013): $\mathrm{Mi}-$ croplastic moves pollutants and additives to worms, reducing functions linked to health and biodiversity. Curr Biol 23(23): 2388-2392.

Burns, E., Boxall, A.B.A. (2018): Microplastics in the aquatic environment: Evidence for or against adverse impacts and major knowledge gaps. Environ Toxicol Chem37:2776-2796.

Cole, M., Lindeque, P., Fileman, E., Halsband, C., Goodhead, R., Moger, J., Galloway, T.S. (2013): Microplastic ingestion by zooplankton. Environ Sci Technol 47:6646-6655

Cole, M., Lindeque, P., Fileman, E., Halsband, C., Galloway, T.S. (2015): The impact of polystyrene microplastics on feeding, function and fecundity in the marine copepod Calanus helgolandicus. Environ Sci Technol 49:1130-1137

Cózar, A., F. Echevarría, J. I. González-Gordillo, X. Irigoien, B. Úbeda, S. Hernández-León, Á. T. Palma, S. Navarro, J. García-de-Lomas and A. Ruiz (2014): Plastic debris in the open ocean. Proceedings of the National Academy of Sciences 111(28): 10239-10244.

ECHA (2011): Leitlinien zu Informationsanforderungen und Stoffsicherheitsbeurteilung Teil B: Ermittlung schädlicher Wirkungen Referenz: ECHA-11-G-09-DE Ausgabedatum: Dezember 2011, Europäische Chemikalienagentur https:// echa.europa.eu/de/guidance-documents/gui dance-on-information-requirements-and-chemical-safety-assessment. (Zugriff 03.07.2020)
ECHA (2016): Guidance on Information Requirements and Chemical Safety Assessment Part E: Risk CharacterisationVersion 3.0 https:// echa.europa.eu/documents/10162/13632/infor mation_requirements part_e en.pdf. (Zugriff 03.07.2020)

ECHA (2018): Note on substance identification and the potential scope of a restriction on uses of "microplastics" Version 1.1-16/10/2018 (Zugriff 05.08.2019) https://echa europa eu/ documents/10162/13641/note_on_substance identification_potential_scope_en.pdf/6f26697e 70b5-9ebe-6b59-2e11085de791,

ECHA (2019): Annex to the annex XV restriction report-Microplastics, version number: 1.2 DATE: 22 August 2019 https://echa.europa.eu/ documents/10162/13641/rest_microplastics_ axvreport_annex_en.pdf/01741d07-f06b-bf328d6f-d6a8de54c4d0 (assessed 23.06.2020)

Eerkes-Medrano, D., R. C. Thompson and D. C. Aldridge (2015): Microplastics in freshwater systems: A review of the emerging threats, identification of knowledge gaps and prioritisation of research needs. Water Research 75: 63-82.

EFSA (2016a): Mikro- und Nanokunststoffe in Lebensmitteln - ein zunehmendes Problem https://www.efsa.europa.eu/de/press/news/ 160623 (Zugriff 05.08.2019)

EFSA (2016b): Statement on the presence of microplastics and nanoplastics in food, with particular focus on seafood. EFSA Journal 2016;14(6):4501, 30 pp. https://doi.org/10. 2903/j.efsa.2016.4501 
Eriksen, M., L. C. Lebreton, H. S. Carson, M. Thiel, C. J. Moore, J. C. Borerro, F. Galgani, P G. Ryan and J. Reisser (2014): Plastic Pollution in the World's Oceans: More than 5 Trillion Plastic Pieces Weighing over 250,000 Tons Afloat at Sea. PLoS One 9(12): el11913.

EU (2018): Communication from the Commission to the European Parliament, the Council, the European economic and social Committee and the Committee of Regions: A European Strategy for Plastics in a Circular Economy. https:// ec.europa.eu/info/research-and-innovation/ research-area/environment/plastics-circulareconomy_en. (Zugriff 05.08.2019)

EurEau (2019): Briefing Note, Microplastics and the water sector-Current knowledge, challenges and possible solutions, 24. April 2019 Everaert, G., Van Cauwenberghe, L., De Rijcke, M., Koelmans, A. A., Mees, J., Vandegehuchte, M., Janssen, C.R. (2018): Risk assessment of microplastics in the ocean: Modelling approach and first conclusions. Environ Pollut242:1930-1938.

Farrell, P., Nelson, K. (2013): Trophic level transfer of microplastics: Mytilus edulis (L.) to Carcinus maenas (L.). Environ Pollut 177:1-3

Gauquie, J., L. Devriese, J. Robbens and B. De Witte (2015): A qualitative screening and quantitative measurement of organic contaminants on different types of marine plastic debris. Chemosphere 138: 348-356.

GESAMP (2015): "Sources, fate and effects of microplastics in the marine environment: a global assessment" (Kershaw, P. J., ed.). (IMO/FAO/ UNESCO-IOC/UNIDO/WMO/IAEA/UN/UNEP/ UNDP Joint Group of Experts on the Scientific Aspects of Marine Environmental Protection). Rep. Stud. GESAMP No. 90, 96 p.

GESAMP (2016): "Sources, fate and effects of microplastics in the marine environment: part two of a global assessment" (Kershaw, P.J., and Rochman, C.M., eds). (IMO/FAO/UNESCOIOC/UNIDO/WMO/LAEA/UN/UNEP/UNDP

Joint Group of Experts on the Scientific Aspects of Marine Environmental Protection). Rep. Stud. GESAMP No. 93, 220 p

Hann, S., C. Sherrington, O. Jamieson, M. Hickman, P. Kershaw, A. Bapasola, G. Cole (2018) Investigating options for reducing releases in the aquatic environment of microplastics emitted by (but not intentionally added in) products, eunomia, Final Report Report for DG Environment of the European Commission

Hidalgo-Ruz, V., L. Gutow, R. C. Thompson, and M. Thiel (2012): Microplastics in the Marine Environment: A Review of the Methods Used for Identification and Quantification, Environmental Science \& Technology 201246 (6), 3060-3075 https://doi.org/10.1021/es2031505

Hohenblum, P., H. Frischenschlager, H. Reisinger, R. Konecny, M. Uhl, S. Mühlegger, H. Habersack, M. Liedermann, P. Gmeiner, B. Weidernhiller, N. Fischer, R. Rindler (2015): Plastik in der Donau. Untersuchungen zum Vorkommen von Kunststoffen in der Donau in Österreich Reports, REP-0547 Umweltbundesamt, Vienna, Austria

Imhof, H. K., C. Laforsch, A. C. Wiesheu, J. Schmid, P. M. Anger, R. Niessner, N. P. Ivleva, (2016): Pigments and plastic in limnetic ecosystems: A qualitative and quantitative study on microparticles of different size classes, Wate Research, Volume 98, Pages 64-74, ISSN 0043 1354, https://doi.org/10.1016/j.watres.2016.03. 015.

Käppler, A., Fischer, D., Oberbeckmann, S. et al (2016): Analysis of environmental microplastics by vibrational microspectroscopy: FTIR, Raman or both? Anal Bioanal Chem 408, 8377-8391. https://doi.org/10.1007/s00216-016-9956-3
Koelmans, A.A., Adil Bakir, G. Allen Burton, and Colin R. Janssen (2016): Microplastic as a Vector for Chemicals in the Aquatic Environment: Critical Review and Model-Supported Reinterpretation of Empirical Studies Environmental Science \& Technology 50 (7), 3315-3326 https://doi.org/ 10.1021 /acs.est.5b06069

Koelmans, A.A., Mohamed Nor, N.H., Hermsen, E., Kooi, M., Mintenig, S.M. and De France, J. (2019): Microplastics in freshwaters and drinking water: Critical review and assessment of data quality. Water Research 155, 410-422. Kosuth, M., S.A. Mason, E. V. Wattenberg (2018): Anthropogenic contamination of tap water, beer and sea salt PLoS One, 13 (4):e0194970. https:// doi.org/10.1371/journal.pone.0194970. eCollection 2018.

Lee, K.W., Shim, W.J., Kwon, O.Y., Kang, J.H. (2013): Size-dependent effects of micro polystyrene particles in the marine copepod Tigriopus japonicus. Environ. Sci. Technol. 47:11278-11283 Li J., H. Liu, J. P. Chen (2018): Microplastics in freshwater systems: A review on occurrence, en vironmental effects, and methods for microplastics detection, Water Research, Volume 137, Pages 362-374, ISSN 0043-1354, https:// doi.org/10. 1016/j.watres.2017.12.056.

Lusher A.L., M. McHugh, R.C. Thompson (2013): Occurrence of microplastics in the gastrointestinal tract of pelagic and demersal fish from the English Channel Mar. Pollut. Bull., 67, pp. 94-99, https://doi.org/10.1016/j.marpolbul. 2012.11.028

Mason, S.A., V. Welch, J. Neratko (2018): Synthetic Polymer Contamination in Bottled Water Fredonia-State University of, New York Miranda, D.A., G.F. Carvalho-Souza (2016): Are we eating plastic-ingesting fish? Mar. Pollut. Bull., 103 pp. 109-114, https://doi.org/10.1016/j. marpolbul.2015.12.035

von Moos, N., Burkhardt-Holm, P., Kohler, A (2012): Uptake and effects of microplastics on cells and tissue of the blue mussel Mytilusedulis L. after an experimental exposure. Environ Sci Technol 46:11327-11335

Murray, F., P.R. Cowie (2011): Plastic contamination in the decapod crustacean Nephrops norvegicus (Linnaeus, 1758) Mar. Pollut. Bull. 62 pp. 1207-1217, https://doi.org/10.1016/j. marpolbul.2011.03.032

Neves, D., P. Sobral, J.L. Ferreira, T. Pereira (2015): Ingestion of microplastics by commercial fish off the Portuguese coast Mar. Pollut. Bull., 101 pp. 119-126, https://doi.org/10.1016/j. marpolbul.2015.11.008

Oehlmann, J., Schulte-Oehlmann, U., Kloas, W., Jagnytsch, O., Lutz, I., Kusk, K.O., Wollenberger, L., Santos, E.M., Paull, G.C., van Look, K.J.W., Tyler, C.R. (2009): Acritical analysis of the biological impacts of plasticizers on wildlife. Phil Trans R Soc B 364:2047-2062

Ory, N.C., Sobral, P., Ferreira, J.L., Thiel, M. (2017): Amberstripe scad Decapterus muroad si (Carangidae) fish ingest blue microplastics resembling their copepod prey along the coast of Rapa Nui (Easter Island) in the South Pacific subtropical gyre. Sci Total Environ 586: 430-437. https://doi.org/10.1016/J.SCITOTENV.2017.01. 175

Prata, J.C., da Costa, J.P., Duarte, A.C. and Rocha-Santos, T. (2019): Methods for sampling and detection of microplastics in water and sediment: A critical review. TrAC Trends in Analytical Chemistry 110, 150-159.

Rani, M., W. J. Shim, G. M. Han, M. Jang, N. A. Al-Odaini, Y. K. Song and S. H. Hong (2015): Qualitative analysis of additives in plastic marine debris and its new products. Archives of environmental contamination and toxicology 69(3):352-366.
Rochman, C., Hoh, E., Kurobe, T. et al. (2013): Ingested plastic transfers hazardous chemicals to fish and induces hepatic stress. Sci Rep 3, 3263 (2013). https://doi.org/10.1038/srep03263

Schwabl, P., B. Liebmann, S. Köppel, P. Königshofer, T. Bucsics, M. Trauner, T. Reiberge (2018): Assessment of microplastic concentrations in human stool-Preliminary results of a prospective study, präsentiert im Rahmen der UEG Week 2018in Wien am 24. Oktober 2018, https://www.meduniwien.ac.at/web/ueberuns/news/detailseite/2018/news-im-oktober2018/erstmals-mikroplastik-im-menschennachgewiesen/ (assessed: 06.07.2020)

Setala, O., Fleming-Lehtinen, V., Lehtiniemi, M. (2014): Ingestion and transfer of microplastics in the planktonic food web. Environ Pollut 185:77-83

Syberg, K., Khan, F.R., Selck, H., Palmqvist, A., Banta, G.T., Daley, J., Sano, L, Duhaime, M.B. (2015): Microplastics: addressing ecological risk through lessons learned. Envtl Toxicol Chem 34:945-953

Talsness, C.E., Andrade, A.J.M., Kuriyama, S.N. Taylor, J.A., vom Saal, F.S. (2009): Component of plastic: experimental studies in animals and relevance for human health. Philos Trans R Soc B 364:2079-2096

Uhl, W. und M. Eftekhardadkhah (2018): Mapping microplastic in Norwegian drinking water, Norwegian Water Report 241, Norwegian Institute for Water Research (NIVA). Camilla Svendsen, Norwegian Institute of Public Health. Norwegian Water BA, Hamar, Norway

UNEP (2016): Marine plastic debris and microplastics: global lessons and research to inspire action and guide policy change. A report prepared for the Second United Nations Environment Assembly. United Nations Environment Programme, Nairobi, $274 \mathrm{pp}$.

Van Cauwenberghe, L. and C. R. Janssen (2014); Microplastics in bivalves cultured for human consumption. Environ Pollut 193: 65-70.

Vendel, A.L., Bessa, F., Alves, V.E.N., Amorim, A.L.A., Patrício, J. and Palma, A.R.T. (2017) Widespread microplastic ingestion by fish assemblages in tropical estuaries subjected to anthropogenic pressures. Marine Pollution Bulletin $117(1), 448-455$.

Vermaire, J.C., C. Pomeroy, S.M. Herczegh, O. Haggart, M. Murphy (2017): Microplastic abundance and distribution in the open water and sediment of the Ottawa River, Canada, and its tributaries Facets, 2 pp. 301-314 https://doi. org/10.1139/facets-2016-0070

Vymetal, U. (2016): Microplastics in bank filtrated Danube Water, Master thesis, University of Natural Resources and Life Sciences, Vienna, Department of Water, Atmosphere and Environment Institute of Sanitary Engineering and Water Pollution Control

Wright, S.L., R.C. Thompson, T.S. Galloway (2013): The physical impacts of microplastics on marine organisms: a review Environ. Pollut., 178 pp. 483-492, https://doi.org/10.1016/j.envpol. 2013.02.031

Ziccardi, L.M., Edgington, A., Hentz, K., Kulacki, K.J. and Kane Driscoll, S. (2016): Microplastics as vectors for bioaccumulation of hydrophobic organic chemicals in the marine environment: A state-of-the-science review. Environmental Toxicology and Chemistry 35(7), 1667-1676.

Hinweis des Verlags Der Verlag bleibt in Hinblick auf geografische Zuordnungen und Gebietsbezeichnungen in veröffentlichten Karten und Institutsadressen neutral. 